\title{
Electrical and thermoelectric transport by variable range hopping in reduced graphene oxide
}

\author{
Min Park, ${ }^{1,2}$ Sung Ju Hong, ${ }^{3}$ Kyung Ho Kim, ${ }^{4}$ Hojin Kang, ${ }^{5}$ Minwoo Lee, ${ }^{6}$ Dae Hong Jeong, ${ }^{6}$ \\ Yung Woo Park, ${ }^{1,5, a)}$ and Byung Hoon $\mathrm{Kim}^{7, \mathrm{a})}$ \\ ${ }^{1}$ Department of Nano Science and Technology, Seoul National University, Seoul 08826, South Korea \\ ${ }^{2}$ Applied Quantum Composites Research Center, Korea Institute of Science and Technology, Wanju 55324, \\ South Korea \\ ${ }^{3}$ Institut für Festkörperphysik, Leibniz Universität Hannover, Appelstraße 2, 30167 Hannover, Germany \\ ${ }^{4}$ Department of Microtechnology and Nanoscience, Chalmers University of Technology, \\ SE-412 96 Gothenburg, Sweden \\ ${ }^{5}$ Department of Physics and Astronomy, Seoul National University, Seoul 08826, South Korea \\ ${ }^{6}$ Department of Chemistry Education, Seoul National University, Seoul 08826, South Korea \\ ${ }^{7}$ Department of Physics and Research Institute of Basic Science, Incheon National University, Incheon 22012, \\ South Korea
}

(Received 8 June 2017; accepted 15 October 2017; published online 26 October 2017)

\begin{abstract}
This study investigated the transport properties of single-layer reduced graphene oxides (rGOs). The rGOs were prepared by the bubble deposition method followed by thermal reduction. The crossover of the transport mechanism from Efros-Shklovskii (ES) variable range hopping (VRH) between the localized states to Mott-VRH was observed near $70 \mathrm{~K}$ using the temperature-dependent conductance. The ES-VRH conduction below $70 \mathrm{~K}$ is apparent in the electric field dependence of the field-driven hopping transport in the high-electric field regime. We also figure out that the thermoelectric power is consistent with the 2D Mott VRH above $70 \mathrm{~K}$. We argue that the VRH conduction results from the topological disorders of $\mathrm{rGO}$ as confirmed by Raman spectroscopy. This infers that the average distance between defects is approximately $2.0 \mathrm{~nm}$. Published by AIP Publishing.

https://doi.org/10.1063/1.4987021
\end{abstract}

The exotic electronic properties of graphene have attracted much attention. ${ }^{1}$ However, the lack of a bandgap has limited the development of graphene based electronic devices. ${ }^{2}$ One of the methods to overcome this limitation is to make defects, which is a useful tool for modifying the electronic structure of graphene. ${ }^{3-5}$ There have been two different approaches to obtain a disordered graphene by artificially controlling the disorder and reduction of graphene oxide $(\mathrm{GO}) .^{6-8} \mathrm{GO}$ is hydrophilic and easily dispersible in water or organic solvents due to the presence of various oxygen functional groups which provide its electrically insulating property and good processability. ${ }^{9}$ The electrical properties of GO can be modified through chemical or thermal reduction. Reduction of GO gradually produces randomly distributed crystalline domains of $s p^{2}$ carbons at nanometer size which are surrounded by the unreduced GO, i.e., the oxygen functional groups. ${ }^{10}$ Although several groups have investigated the charge transport properties of reduced GO (rGO), there is still some disagreement among their results. They provided different explanations for the charge transport behaviors of rGO, e.g., Mott variable range hopping (VRH) ${ }^{11,12}$ Efros-Shklovskii (ES)-VRH, ${ }^{13}$ and thermal activation, ${ }^{14}$ because these results were obtained with different sample geometries and different temperature ranges. However, the study of the charge transport mechanism of rGO is indispensable for not only understanding its fundamental properties but also realizing its industrial applications. Therefore, the probing transport mechanism of rGO

\footnotetext{
${ }^{\text {a) }}$ Authors to whom correspondence should be addressed: ywpark@phya.snu. ac.kr and kbh37@incheon.ac.kr.
}

is an important subject in the field of graphene-based electronics.

In this study, we report the temperature dependence of conductance $[G(T)]$ and thermoelectric power (TEP) measurements as a function of the gate voltage $\left(V_{g}\right)$ in a large area single layer rGO. The $G(T)$ follows the ES-VRH model in the full range of $V_{g}$. The TEP shows that the two dimensional (2D) Mott VRH is the main transport mechanism at $T>50 \mathrm{~K}$.

A large-area GO was synthesized by the modified Hummers method using natural expandable graphite. ${ }^{15} \mathrm{~A}$ single-layer GO was deposited onto a $\mathrm{SiO}_{2}(285 \mathrm{~nm}) / \mathrm{Si}$ substrate by the bubble deposition method. ${ }^{16}$ The rGO was prepared by thermal annealing in a $\mathrm{H}_{2} / \mathrm{Ar}$ flow at $1000^{\circ} \mathrm{C}$ for $6 \mathrm{~h}$. Afterwards, the rGO was transferred onto the $\mathrm{SiO}_{2}(285 \mathrm{~nm}) /$ $\mathrm{Si}$ substrate with a pre-patterned alignment marker. The single-layer rGO was selected by optical contrast using an optical microscope and an atomic force microscope (AFM, Asylum Research). Rectangular-shaped rGO was defined by conventional electron-beam (e-beam) lithography (MIRA3, Tescan) and oxygen plasma etching. The electrodes and micro-heater were fabricated by e-beam lithography followed by thermal evaporation $(\mathrm{Cr} / \mathrm{Au}=5 / 50 \mathrm{~nm})$ and using a lift-off process. The transport properties of the rGO device were studied in two-terminal configurations. All measurements were performed in a $14 \mathrm{~T}$ superconducting magnet (Oxford). The $V_{g}$-dependent resistance and TEP were measured by the DC method using a semiconductor parameter analyzer (4200SCS, Keithley). The rGO was also characterized by X-ray photoelectron spectroscopy [XPS, PHI 5000 Versa Probe, $\operatorname{Phi}(\Phi)]$ and Raman spectroscopy $(532 \mathrm{~nm}$ wavelength with a power of $10 \mu \mathrm{W}$, JY-Horiba). 
The single-layer rGO had a $1 \mathrm{~nm}$ thickness of the rGO determined by AFM shown in the inset of Fig. 1(a). Figure 1(a) displays that the elemental composition of the rGO was investigated by XPS. We found that the amount of C-C and $\mathrm{C}=\mathrm{C}$ species increased up to $82 \%$. We carried out the Raman spectroscopy measurement to verify the quality of rGO. The Raman spectra were changed significantly after the reduction process. The intensity of the $G$ peak decreased compared with the intensity of the $D$ peak, and the $D^{\prime}$ peak near $1620 \mathrm{~cm}^{-1}$ was slightly developed as shown in Fig. 1(b). The ratios of $I_{D} / I_{G}$ indicate the degree of disorder in graphene-based materials. ${ }^{17}$ The $I_{D} / I_{G}$ ratio increased from 0.93 to 1.87 upon reduction, which indicates a decrease in the average size but an increase in the number of $s p^{2}$ domains upon reduction. According to the curve associated with the $I_{D} / I_{G}$ ratio and the distance between two defects $\left(L_{D}\right)$ studied, ${ }^{18}$ the $I_{D} / I_{G}$ ratio and the full width at halfmaximum (FWHM) indicate the distance between the two defects. The FWHM of the $G\left(55 \mathrm{~cm}^{-1}\right), D\left(46 \mathrm{~cm}^{-1}\right)$, and $2 D\left(110 \mathrm{~cm}^{-1}\right)$ peaks after thermal reduction corresponds to carbon materials in a topologically disordered state where the $L_{D}$ of the rGO is approximately $2.0 \mathrm{~nm}$.

Figure 1(c) shows the representative transfer curve as a function of the temperature in the temperature range of $10<T<300 \mathrm{~K}$, with a source-drain voltage $\left(V_{D S}\right)$ of $10 \mathrm{mV}$ for which the current-voltage $(I-V)$ curves are Ohmic. The $V_{g}$-dependent conductance exhibited ambipolar transport over the entire temperature range. The temperature dependence of conductance $G(T)$ as a function of $V_{g}$ decreased as the temperature decreased [Fig. 1(d)], which probably originates from the characteristic of the strongly localized regime of the VRH. As expected, $G(T)$ follows the conventional Mott-VRH equation: $G(T)=G_{0} \exp \left(-T_{0} / T\right)^{p}$, where $G_{0}$ is the conductance at infinite temperature and $T_{0}$ is the Mott characteristic temperature. The exponent $p$ depends on the (a)
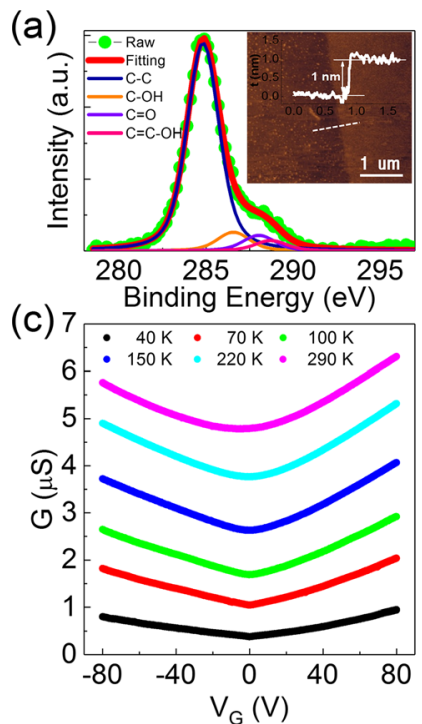

(b)

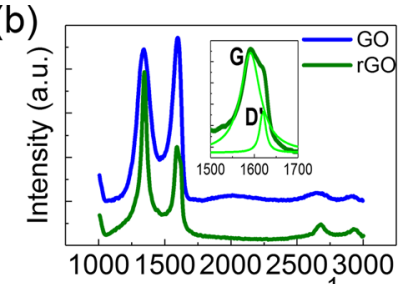

(d)

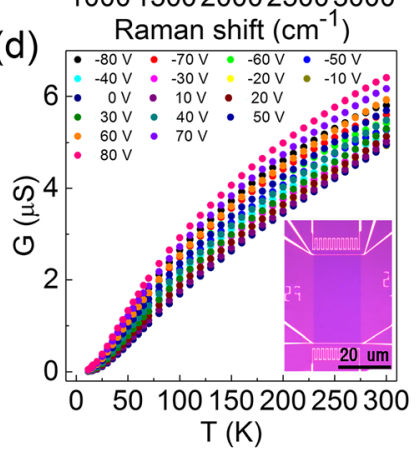

FIG. 1. (a) XPS spectra for rGO with deconvolution of the $\mathrm{C} 1 \mathrm{~s}$ peaks. The inset shows the AFM image of an rGO device. (b) Raman spectra before (blue curve) and after reduction (green curve). Inset: magnified region for the $G$ peak. (c) The $V_{g}$ dependence of conductance with a fixed drain voltage of $10 \mathrm{mV}$ at different temperatures. (d) $G(T)$ as a function of temperature in the range of $10<\mathrm{T}<300 \mathrm{~K}$. The inset displays an optical image of the device. dimension of the systems or the shape of the density of state (DOS) ${ }^{19}$ Note that the Mott-VRH does not include the Coulomb interaction between the localized states. When coulomb interactions exist in the VRH system, the DOS near the Fermi level diminishes because a soft gap is created in the vicinity of the Fermi level called the Coulomb gap (CG). ${ }^{20}$ Therefore, the hopping equation is modified by the ES-VRH model as follows: $G(T)=G_{0} \exp \left(-T_{E S} / T\right)^{1 / 2}$, with $T_{E S}$ $=\beta e^{2} / 4 \pi \varepsilon \varepsilon_{0} k_{B} \xi$, where $\beta$ is a numerical constant with a value of 6.2 in $2 \mathrm{D},{ }^{20} \xi$ is the localization length, $\varepsilon$ is the effective dielectric constant, $\varepsilon_{0}$ is the vacuum permittivity, $k_{B}$ is the Boltzmann constant, and $e$ is the electron charge. In the case of the ES-VRH, $p=1 / 2$ is irrespective of the dimensions. The determination of the exponent $(p)$ unambiguously distinguishes between the two different VRH mechanisms. For that purpose, we used a method based on the logarithmic derivation of the reduced activation energy $(W)$ introduced by Zabrodskii and Zinoveva. ${ }^{21} W$ is expressed by the following equation: $W(T)=\partial \ln G / \partial \ln T=p\left(T_{0} / T\right)^{p}$, where the value of $p$ can be obtained from the slope of $\ln W$ versus $\ln$ $T$. Figure 2(a) shows $W \mathrm{~s}$ at various temperatures on a $\log$ scale for which the experimental data were plotted as a function of $V_{g}$. The linear solid violet line and pink line correspond to $p=1 / 2$ and $p=1 / 3$, respectively. The ES-VRH conduction $(p=1 / 2)$ is dominant at low temperature; however, the 2D Mott -VRH behavior $(p=1 / 3)$ becomes prominent at high temperature. When the thermal energy is higher than the Coulomb gap energy $\left(\mathrm{k}_{\mathrm{B}} \mathrm{T} \gg \mathrm{E}_{\mathrm{CG}}\right),{ }^{22}$ the Coulomb interaction becomes negligible. As a result, the charge transport is not affected by ES-VRH anymore, and the Mott-VRH conduction recovered. It is difficult to distinguish an accurate crossover temperature from Fig. 2(a). Thus, we plotted $G$ vs $T^{-1 / 2}$ and $T^{-1 / 3}$ for all $V_{g}$ s. Figure 2(b) shows the conductance at $V_{g}=0$ as a function of $T^{-1 / 2}$ (red square dot) and $T^{-1 / 3}$ (blue square dot) (The other plots for various $V_{g}$ values are plotted in Fig. S1). The linear fittings of $\ln G$ to $T^{-1 / 2}$ and $T^{-1 / 3}$ are plotted as a solid red line and a solid blue line, respectively. $\ln G$ to $T^{-1 / 2}$ is well fitted at low temperatures and then deviates near $65-70 \mathrm{~K}$. In contrast, the fitting of $\ln G$ to $T^{-1 / 3}$ is well matched in high temperature regimes. However, it deviates near $70 \mathrm{~K}$. It is worth noting that the deviation temperature is almost the same. It means that the crossover occurs at a crossover temperature $\left(T_{C O}\right)$ of approximately $70 \mathrm{~K}$ shown in Fig. 2(c). The ES-VRH and 2D MottVRH behaviors of $G(T)$ for each temperature regime are shown in Figs. S2(a) and S2(b), respectively.

The Coulomb gap energy, $E_{C G}=T_{E S} / \beta \sqrt{4 \pi}$, was calculated to validate $T_{C O}$, in which $T_{E S}$ is obtained from the linear fitting of Fig. S3(a). Figure 2(d) shows both $E_{C G}$ and $\xi$ values as a function of $V_{g} . E_{C G}$ and $\xi$ are $32 \mathrm{~K}$ and $19.3 \mathrm{~nm}$ at a $V_{g}=0$, and $E_{C G}$ decreases as the magnitude of $V_{g}$ increases. The smaller $E_{C G}$ compared with $T_{C O}$ is obvious because the thermal energy will be larger than $E_{C G}$ for the occurrence of crossover. $E_{C G}$ at a $V_{g}$ of 0 is suppressed by increasing positive and negative gate voltage, which arises from the increase in overlapping wave functions for localized electrons. The increase in the overlap enlarges the domains of the delocalized electrons and presumably screens the Coulomb interaction. ${ }^{23}$ The reduction of $E_{C G}$ at high 

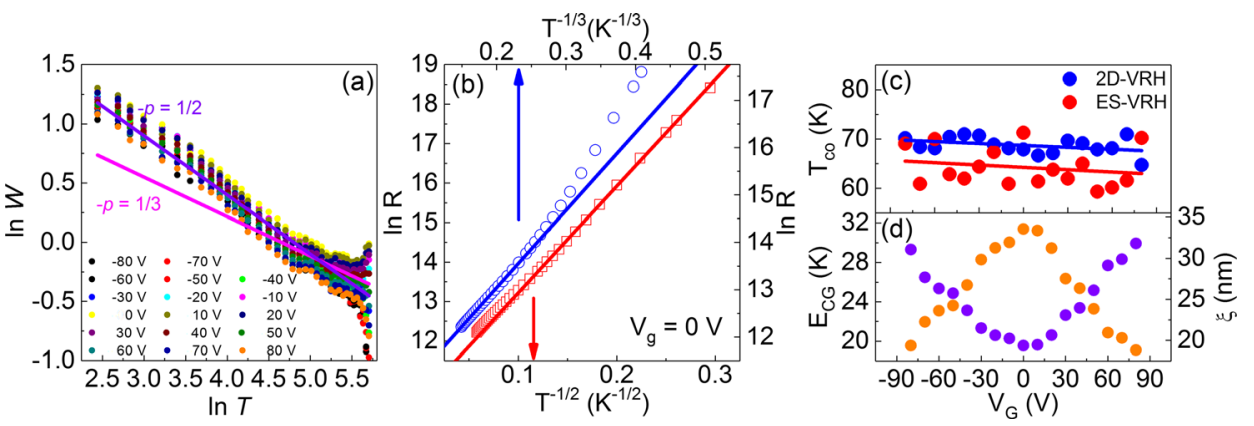

FIG. 2. (a) Reduced activation energy as a function of temperature on a log$\log$ scale for $V_{g}$. (b) Semi-log scale plot of $\ln G(T) \propto T^{-1 / 2}$ and $\ln G(T)$ $\propto T^{-1 / 3}$ for $V_{g}=0$. The symbols represent experimental data, and the solid lines represent a linear fit to $T^{-1 / 2}$ (red line) and $T^{-1 / 3}$ (blue line). (c) The crossover temperature as a function of $V_{g}$ calculated from Fig. S1. (d) The $V_{g}$ dependence of $E_{C G}$ (orange dots) and $\xi$ (violet dots). carrier densities and the switching of the ES-VRH to the Mott-VRH have been reported in graphene antidot lattices. ${ }^{24}$

The ES-VRH behavior was further confirmed by the electric field dependence of conductance at high electric fields. Figure 3(a) shows $G(\mathrm{E})$ at $1.6 \mathrm{~K}$ as a function of $V_{g}$. The electric field-dependent $G(T)$ is strongly suppressed in a high electric field regime at a low temperature as follows: $:^{25}$ $G(E) \sim \exp \left(-E_{0} / E\right)^{1 / 2}$, with $E_{0}=k_{B} T_{E S} / 2 e \xi$, where $G(E)$ is the non-Ohmic conductance $(d I / d V)$. This electric field dependence is expected in the ES-VRH above the critical electric field $\left(E_{C} \gg 2 k_{B} T / e \xi\right){ }^{25,26} E_{0}$ decreases as the carrier density increases [Fig. 3(b)]; this behavior is consistent with that of $E_{C G}$ obtained in the Ohmic regime of the lowelectric field.

We further confirm the ES-VRH by magnetoresistance (MR). The negative MR is observed and follows the ESVRH, which is interpreted by the quantum interference effect in the VRH system (see the MR of rGO in the supplementary material).

Figure 4(a) shows the $V_{g}$ dependence of the TEP $(S)$ as a function of the temperature $(50 \mathrm{~K}<T<300 \mathrm{~K})$. Typically, the sign of the TEP of graphene is switched across the charge neutrality point (CNP). In addition, the sign of the TEP is very sensitive to the kind of majority carrier. ${ }^{27}$ The positive and negative values of the TEP indicate that holes $(S>0)$ and electrons $(S<0)$ are the majority charge carriers, respectively. We observed that the magnitude of the TEP increased with the increase in $V_{g}$ regardless of the carrier type. It was reported that the TEP increased with an increase in $V_{g}$ near the Dirac point in graphene, which was interpreted by the result from the electron and hole puddle effect near the Dirac point. ${ }^{27}$ Moreover, the broadening of the peak to the dip width in $S\left(V_{g}\right)$ was observed in the low mobility graphene, ${ }^{28-30}$ which is also related to the electron and hole puddle effect due to the disorder effect and charged impurity
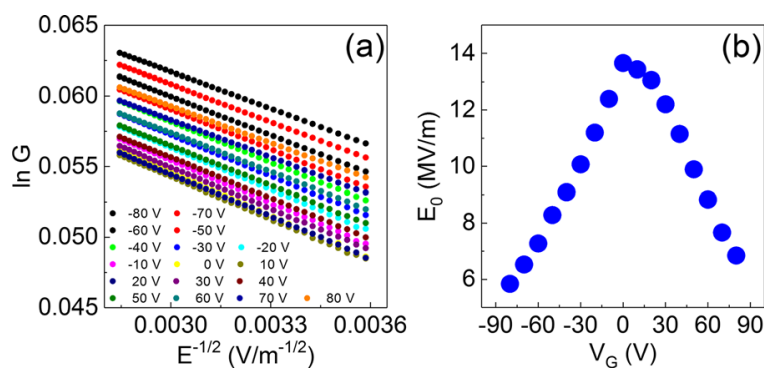

FIG. 3. $G(E)$ as a function of gate voltage. (a) Semi $\log$ plot of $\ln G(E)$ $\propto E^{-1 / 2} \ln$ for the rGO device at $1.6 \mathrm{~K}$. (b) $E_{0}$ obtained from the fitting of $G(E) \propto \exp \left(-E_{0} / E\right)^{1 / 2}$. in the graphene. In this regime, the compensated TEP can be expressed as a simplified two-component model with the carrier density of the electron $\left(n_{e}\right)$ and hole $\left(n_{h}\right)$ as follows: $S=S^{\prime}\left(\left|n_{h}\right|-\left|n_{e}\right|\right) /\left(\left|n_{h}\right|+\left|n_{e}\right|\right),{ }^{31}$ where the TEP increases from zero at the complete compensation as the density of the majority carrier exceeds that of the minority carrier. Then, the magnitude of the TEP decreases as the Fermi energy moves to degenerated states. However, the localized states due to the defects, impurities, and electron-hole puddle are formed in a broad bandwidth in the rGO, for which we observed the ES-VRH behaviors. Thus, the maximum value of the TEP is far from the CNP, which is not accessible in our $V_{g}$ range.

Figure 4(b) shows the temperature-dependent TEP, $S(T)$, with respect to $V_{g}$. The TEP of the rGO follows the 2D Mott VRH which is linear with respect to $T^{1 / 3}$. The temperature
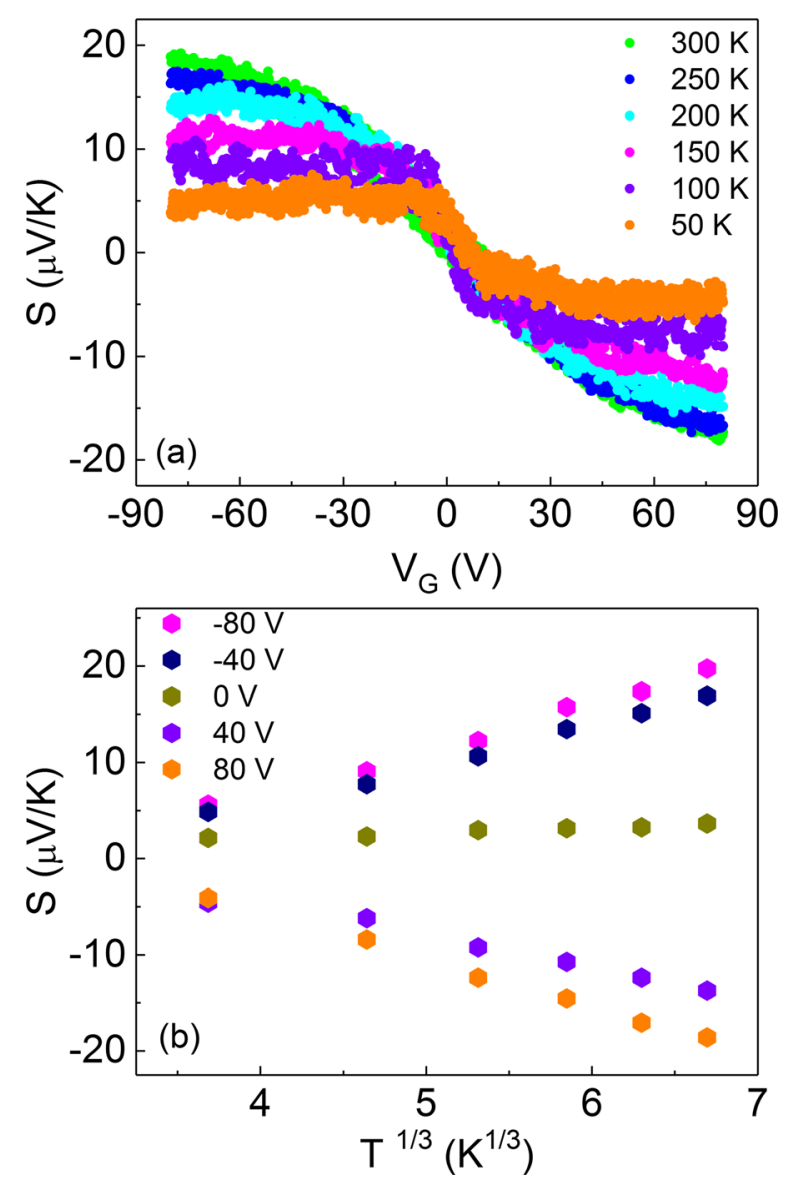

FIG. 4. (a) TEP as a function of $V_{g}$ for $\mathrm{T}=50,100,150,200,250$, and $300 \mathrm{~K}$. (b) $S(T) \propto T^{1 / 3}$ for $V_{g}=-80,-40,0,40$, and $80 \mathrm{~V}$. 
dependence of the TEP in the Mott VRH is expressed as follows: ${ }^{32}$

$$
S(T)_{M o t t-V R H} \approx k_{B} T\left(\frac{T_{M}}{T}\right)^{\frac{2}{d+1}} \times \frac{d n\left(E_{F}\right)}{d E_{F}} \propto T^{\frac{d-1}{d+1}}
$$

where $n(E)$ is the density of the states and $d$ is the dimensionality of the system. As mentioned above, we observed the 2D Mott VRH behavior in $G(T)$ within the temperature range $(70 \mathrm{~K}<\mathrm{T}<300 \mathrm{~K})$. The behavior of both $G(T)$ and $S(T)$ indicates that the 2D VRH is the dominant conduction mechanism above $70 \mathrm{~K}$. The ES-VRH is prominent at low temperatures at which the thermal energy is smaller than the Coulomb gap. Furthermore, $E_{C G}$ estimated from the temperature dependence of conductance was $32 \mathrm{~K}$. Therefore, we conclude that the ES-VRH is dominant below $70 \mathrm{~K}$, and the transition to Mott's 2D VRH occurs at about $70 \mathrm{~K}$. It is worth noting that crossover exists in the charge transport phenomenon of rGO and that the energy for the crossover is about $70 \mathrm{~K}$.

In summary, we investigate the electrical and thermoelectric transport of rGO. $G(T)$ with gate voltages of $-80 \mathrm{~V}<V<80 \mathrm{~V}$ follows the ES-VRH for which the size of the Coulomb gap decreases as the carrier density increases. The $V_{g}$ dependence of the TEP indicates the broad bandwidth of the localized states. The ES-VRH is dominant below a $T$ of $70 \mathrm{~K}$, and the $2 \mathrm{D}-\mathrm{VRH}$ is the main transport mechanism at temperatures in the range of $70<\mathrm{T}<300 \mathrm{~K}$ confirmed by the temperature dependence of the TEP.

See supplementary material for additional figures of $\mathrm{G}(T)$ and MR of rGO at various temperatures.

This work was supported by the Swedish-Korean Basic Research Cooperative Program (No. NRF2014R1A2A1A12067266) of the National Research Foundation of Korea (NRF) and by the FPRD of BK21 plus through the MEST, Korea. This work was supported by the Korea Institute of Science and Technology (KIST) Institutional Program. B.H.K. acknowledges the support from NRF (No. NRF-2017R1A1A1A05000789) and Basic Science Research Program through NRF funded by the Ministry of Education (No. NRF-2017R1A6A1A06015181).

${ }^{1}$ K. S. Novoselov, A. K. Geim, S. Morozov, D. Jiang, M. Katsnelson, I. Grigorieva, S. Dubonos, and A. Firsov, Nature 438(7065), 197-200 (2005).

${ }^{2}$ M. Y. Han, B. Özyilmaz, Y. Zhang, and P. Kim, Phys. Rev. Lett. 98(20), 206805 (2007).

${ }^{3}$ J. Haskins, A. Kınacı, C. Sevik, H. Sevinçli, G. Cuniberti, and T. Çağın, ACS Nano 5(5), 3779-3787 (2011).

${ }^{4}$ R. Balog, B. Jørgensen, L. Nilsson, M. Andersen, E. Rienks, M. Bianchi, M. Fanetti, E. Lægsgaard, A. Baraldi, S. Lizzit, Z. Slijvancanin, F.
Besenbacher, B. Hammer, T. G. Pedersen, P. Hofmann, and L. Hornekaer, Nat. Mater. 9(4), 315-319 (2010).

${ }^{5}$ D. Haberer, D. Vyalikh, S. Taioli, B. Dora, M. Farjam, J. Fink, D. Marchenko, T. Pichler, K. Ziegler, M. S. Dresselhaus, M. Knupfer, B. Buchner, and A. Gruneis, Nano Lett. 10(9), 3360-3366 (2010).

${ }^{6}$ D. Teweldebrhan and A. A. Balandin, Appl. Phys. Lett. 94(1), 013101 (2009).

${ }^{7}$ I. Childres, L. A. Jauregui, J. Tian, and Y. P. Chen, New J. Phys. 13(2), 025008 (2011).

${ }^{8}$ P. Willke, J. A. Amani, A. Sinterhauf, S. Thakur, T. Kotzott, T. Druga, S. Weikert, K. Maiti, H. Hofsäss, and M. Wenderoth, Nano Lett. 15(8), 5110-5115 (2015).

${ }^{9}$ Y. W. Zhu, S. Murali, W. W. Cai, X. S. Li, J. W. Suk, J. R. Potts, and R. S. Ruoff, Adv. Mater. 22(35), 3906-3924 (2010).

${ }^{10}$ E. C. Mattson, H. H. Pu, S. M. Cui, M. A. Schofield, S. Rhim, G. H. Lu, M. J. Nasse, R. S. Ruoff, M. Weinert, M. Gajdardziska-Josifovska, J. H. Chen, and C. J. Hirschmugl, ACS Nano 5(12), 9710-9717 (2011).

${ }^{11}$ C. Gómez-Navarro, R. T. Weitz, A. M. Bittner, M. Scolari, A. Mews, M. Burghard, and K. Kern, Nano Lett. 7(11), 3499-3503 (2007).

${ }^{12}$ A. B. Kaiser, C. Gómez-Navarro, R. S. Sundaram, M. Burghard, and K. Kern, Nano Lett. 9(5), 1787-1792 (2009).

${ }^{13}$ D. Joung and S. I. Khondaker, Phys. Rev. B 86(23), 235423 (2012).

${ }^{14}$ S. Wang, P.-J. Chia, L.-L. Chua, L.-H. Zhao, S. Sivaramakrishnan, M. Zhou, R. G.-S. Goh, R. H. Friend, A. T.-S. Wee, and P. K.-H. Ho, Adv. Mater. 20(18), 3440-3446 (2008).

${ }^{15}$ D. Li, M. B. Müller, S. Gilje, R. B. Kaner, and G. G. Wallace, Nat. Nanotechnol. 3(2), 101-105 (2008).

${ }^{16} \mathrm{~J}$. Azevedo, C. Costa-Coquelard, P. Jegou, T. Yu, and J. J. Benattar, J. Phys. Chem. C 115(30), 14678-14681 (2011).

${ }^{17}$ A. C. Ferrari, Solid State Commun. 143(1), 47-57 (2007).

${ }^{18}$ L. G. Cançado, A. Jorio, E. M. Ferreira, F. Stavale, C. Achete, R. Capaz, M. Moutinho, A. Lombardo, T. Kulmala, and A. Ferrari, Nano Lett. 11(8), 3190-3196 (2011).

${ }^{19}$ N. F. Mott and E. A. Davis, Electronic Processes in Non-Crystalline Materials, 2nd ed. (Clarendon Press, Oxford University Press, Oxford, New York, 1979).

${ }^{20}$ B. I. Shklovskiı and A. L. Éfros, Electronic Properties of Doped Semiconductors (Springer-Verlag, Berlin, New York, 1984).

${ }^{21}$ A. G. Zabrodskii and K. N. Zinov'eva, Zh. Eksp. Teor. Fiz. 86, 727-742 (1984), available at http://www.jetp.ac.ru/cgi-bin/e/index/e/59/2/ p425? a $=$ list.

${ }^{22}$ H. Liu, A. Pourret, and P. Guyot-Sionnest, ACS Nano 4(9), 5211-5216 (2010).

${ }^{23}$ A. V. Dvurechenskii and A. I. Yakimov, Zh. Eksp. Teor. Fiz. 95, 159-169 (1989), available at http://www.jetp.ac.ru/cgi-bin/e/index/r/95/1/ p159?a $=$ list.

${ }^{24}$ A. Giesbers, E. Peters, M. Burghard, and K. Kern, Phys. Rev. B 86(4), 045445 (2012).

${ }^{25}$ D. Yu, C. Wang, B. L. Wehrenberg, and P. Guyot-Sionnest, Phys. Rev. Lett. 92(21), 216802 (2004)

${ }^{26}$ K. H. Kim, S. Lara-Avila, H. Kang, H. He, J. Eklöf, S. J. Hong, M. Park, K. Moth-Poulsen, S. Matsushita, K. Akagi, S. Kubatkin, and Y. W. Park, Sci. Rep. 6, 37783 (2016).

${ }^{27}$ Y. M. Zuev, W. Chang, and P. Kim, Phys. Rev. Lett. 102(9), 096807 (2009).

${ }^{28}$ J. Duan, X. Wang, X. Lai, G. Li, K. Watanabe, T. Taniguchi, M. Zebarjadi, and E. Y. Andrei, Proc. Natl. Acad. Sci. U.S.A. 113(50), 14272-14276 (2016).

${ }^{29}$ P. Wei, W. Bao, Y. Pu, C. N. Lau, and J. Shi, Phys. Rev. Lett. 102(16), 166808 (2009).

${ }^{30}$ X. Liu, D. Wang, P. Wei, L. Zhu, and J. Shi, Phys. Rev. B 86(15), 155414 (2012).

${ }^{31}$ E. Hwang, E. Rossi, and S. D. Sarma, Phys. Rev. B 80(23), 235415 (2009).

${ }^{32}$ M. J. Burns and P. M. Chaikin, J. Phys. C: Solid State Phys. 18(24), L743 (1985). 